\title{
Platform Competition with Intra-Group Externalities
}

\author{
Barna Bakó* Daniel Fatay ${ }^{\dagger}$
}

December 10, 2015

\begin{abstract}
In this article we analyze asymmetric two-sided markets. Two types of agents are assumed to interact with each other and we assume that agents of one type derive utility from inter-group interactions, while the other type of agents benefit from intra-group rather than from inter-group interactions as it is assumed in the standard symmetric two-sided markets model. First, we consider a monopoly platform, then we analyze competing platforms, both with single-homing and multi-homing abilities.
\end{abstract}

\section{Introduction}

According to Rochet and Tirole (2003) many markets with network externalities are twosided. These markets are characterized with two distinct sides whose benefits originate from being able to interact with each other through a common platform. Good examples abound from credit cards, computer operating systems and video game consoles to job requirement sites and dating agencies. The core concept behind the model of the two sided markets is that the utility obtained by each side of the market is crucially affected by the size of the other side of the market. Credit card holders, for example, gain positive network effect if their card is widely accepted and merchants that accepts the card are affected positively if the number of card holders increase.

Although the topic of two-sided markets is well researched (see e.g. Rochet and Tirole, 2003; Armstrong and Wright, 2006; Armstrong, 2006; Weyl, 2010; Hagiu and Halaburda, 2014) to the best of our knowledge little attention has been paid to the asymmetric case

\footnotetext{
*MTA-BCE 'Lendúlet' Strategic Interactions Research Group, Corvinus University of Budapest, Department of Microeconomics, Fővám tér 8, E225-A, Budapest, 1093, Hungary, e-mail: barna.bako@uni-corvinus.hu

${ }^{\dagger}$ KUKA Robotics
} 
of such markets. A good example for an asymmetric two-sided market would be the social media. While it is reasonable to assume that the number of users of a social media crucially affects the utility gained by the advertisers, the complementary assumption related to the network effect caused by the advertisers is perhaps secondary. Users most likely join the social media to be able to interact with their peers rather than to interact with the advertisers. If this is the case, such markets must be modelled with this asymmetric network effect in mind since the original symmetric two-sided models might lead to inaccurate deductions.

In this article we analyze asymmetric two-sided markets. Two types of agents are assumed to interact with each other and we assume that agents of type 1 derive utility from inter-group interactions, while agents of type 2 benefit from intra-group rather than from inter-group interactions as it is assumed in the standard symmetric two-sided markets model. First, we consider a monopoly platform, then we analyze competing platforms, both with single-homing and multi-homing abilities.

\section{The Model}

\subsection{A Monopoly Platform}

Suppose there are two type of agents $(i=1,2)$ served by an intermediary platform. A member of group 1 benefits from interactions with agents of its own group, while a member of group 2 cares about the number of members from the opposite group who use the platform. ${ }^{1}$ That is, we assume that agents of type 1 derive utility from intra-group interactions rather then from inter-group interactions as it is assumed in the standard symmetric twosided markets model, while, agents of type 2 benefit from inter-group interactions. More specifically, we assume that the utilities of the agents can be given as

Assumption $1 u_{1}=\alpha_{1} n_{1}-p_{1}$ and $u_{2}=\alpha_{2} n_{1}-p_{2}$

where $p_{i}(i=1,2)$ is the price charged by the platform to side $i, \alpha_{i}$ measures the benefit an agent of side $i$ derives from interacting with an agent of type 1 if the platform attracts $n_{1}$ members of the type 1 . To keep the model as general as possible we allow $p_{i}<0$ whenever is feasible. For example, $p_{1}<0$ means that the platform charges negative price to side 1 in order to exploit the network effect users of that side create.

We define demands as functions of utilities offered by the platform to each type of agent rather than prices and in order to close the model we assume that ${ }^{2}$

Assumption $2 n_{i}=\Phi_{i}\left(u_{i}\right)$

\footnotetext{
${ }^{1}$ For example, one may think of group 1 as the users of a social media platform and group 2 as firms who want to advertise on the platform. Obviously users derive benefit from being able to interact with more users, while an advertiser ultimately cares only about the number of users who use the platform.

${ }^{2}$ Modeling demands as functions of utilities has the consequence that prices are implicitly determined by equations given in Assumption 1 .
} 
where $\Phi_{i}\left(u_{i}\right)$ is an increasing function of $u_{i}$. That is, we assume that if the utility offered by the platform to side $i$ is $u_{i}$ a number of $n_{i}$ members will join side $i$ of the platform. Furthermore, we assume that in order to serve a member of group $i$ the platform experiences a $c_{i}$ unit cost and for the sake of tractability we assume that the platform's fix costs are equal to zero. More formally

Assumption $3 C\left(n_{1}, n_{2}\right)=\sum_{i=1}^{2} n_{i} c_{i}$

Based on these assumptions the platform's profit function $\left(\pi=\sum_{i=1}^{2} n_{i}\left(p_{i}-c_{i}\right)\right)$ in terms of utilities can be given as follows

$$
\pi\left(u_{1}, u_{2}\right)=\Phi_{1}\left(u_{1}\right)\left[\alpha_{1} \Phi_{1}\left(u_{1}\right)-u_{1}-c_{1}\right]+\Phi_{2}\left(u_{2}\right)\left[\alpha_{2} \Phi_{1}\left(u_{1}\right)-u_{2}-c_{2}\right]
$$

The first-order conditions for the platform's optimal pricing problem are

$$
\begin{aligned}
\frac{\partial \pi\left(u_{1}, u_{2}\right)}{\partial u_{1}} & =\Phi_{1}^{\prime}\left(u_{1}\right)\left[\alpha_{1} \Phi_{1}\left(u_{1}\right)-u_{1}-c_{1}\right]+\Phi_{1}\left(u_{1}\right)\left[\alpha_{1} \Phi_{1}^{\prime}\left(u_{1}\right)-1\right] \\
& +\Phi_{2}\left(u_{2}\right) \alpha_{2} \Phi_{1}^{\prime}\left(u_{1}\right)=0 \\
\frac{\partial \pi\left(u_{1}, u_{2}\right)}{\partial u_{2}} & =\Phi_{2}^{\prime}\left(u_{2}\right)\left[\alpha_{2} \Phi_{1}\left(u_{1}\right)-u_{2}-c_{2}\right]-\Phi_{2}\left(u_{2}\right)=0
\end{aligned}
$$

where $\Phi_{i}^{\prime}\left(u_{i}\right)$ stands for the first order derivative of $\Phi_{i}\left(u_{i}\right)$. Rewriting these expressions, using Assumption 1, we have that:

Proposition 1 In asymmetric two-sided markets a monopolist platform's profit-maximizing prices satisfy

$$
\begin{aligned}
p_{1} & =c_{1}-\alpha_{1} n_{1}-\alpha_{2} n_{2}+\frac{\Phi_{1}\left(u_{1}\right)}{\Phi_{1}^{\prime}\left(u_{1}\right)} \\
p_{2} & =c_{2}+\frac{\Phi_{2}\left(u_{2}\right)}{\Phi_{2}^{\prime}\left(u_{2}\right)}
\end{aligned}
$$

Hence, the platform charges a price for group 1 equal to the marginal cost adjusted with the economical value of the benefits an agent creates on each side of the platform and with a factor related to the elasticity of demand.

Definition 1 Let $\eta_{1}\left(p_{1} \mid n_{1}\right) \equiv \frac{p_{1} \Phi_{1}^{\prime}\left(u_{1}\right)}{\Phi_{1}\left(u_{1}\right)}$ and $\eta_{2}\left(p_{2} \mid n_{1}\right) \equiv \frac{p_{2} \Phi_{2}^{\prime}\left(u_{2}\right)}{\Phi_{2}\left(u_{2}\right)}$ denote the price elasticities of demand given that the platform attracts $n_{i}$ members on side $i$ (where $\left.i=1,2\right){ }^{3}$

Using this definition from the expressions given in Proposition 1 we have that the platform's profit maximizing prices satisfy

\footnotetext{
${ }^{3}$ To simplify notation generally we do not write out the independent variables of $\eta_{i}(\cdot)$ and we will mention them only when it is necessary to prevent misunderstanding.
} 


\section{Corollary 1}

$$
\begin{aligned}
\frac{p_{1}-c_{1}}{p_{1}} & =\frac{1}{\eta_{1}}-\frac{\alpha_{1} n_{1}+\alpha_{2} n_{2}}{p_{1}} \\
\frac{p_{2}-c_{2}}{p_{2}} & =\frac{1}{\eta_{2}}
\end{aligned}
$$

The second expression is similar to the familiar Lerner formula for monopoly pricing and as a consequence $p_{2}>0$ in equilibrium, however, the mark-up realized on group 1 is lower than the one in standard monopoly pricing. A monopoly platform might be willing to sacrifice somewhat its margin earned on group 1 or even to set a price lower than the marginal cost in order to motivate agents of type 1 to join the platform which makes the platform more attractive to members of both groups, which ultimately may increase its profit. This may happen when the price elasticity of group 1 is high or when the benefit caused by the presence of a group 1 member either on its own group or on the other group is large. Furthermore, notice that in equilibrium the platform will always set a price $p_{2}$ such that $\eta_{2}>1$. Interestingly, the margin realized on group 2 and ultimately the price set to this group explicitly does not depend on the number of members who join the platform from group 1, even though their utility is solely derived from the ability to interact with them. However, there is an implicit effect as agents of group 1 affect the price set by the platform on group 2. As more agents of type 1 are attracted to the platform the utility of each member of group 2 is increasing and such the platform gets more room to set a higher price.

Corollary $2 p_{1}<p_{2}$ if the price elasticity of group 1 is greater than the one of group 2 , i.e. $\eta_{1}>\eta_{2}$, and/or the marginal cost to serve group 1 is significantly smaller than the one of group 2, i.e. $c_{1}<c_{2}$, and/or the benefit enjoyed by the platform's users caused by a member of group $1\left(\alpha_{1} n_{1}+\alpha_{2} n_{2}\right)$ is large. Moreover, this may happen even if $\eta_{1}<1$ and/or $c_{1}>c_{2}$ and/or $\alpha_{1} n_{1}+\alpha_{2} n_{2}$ is small.

The optimal profit of the monopolist platform can be calculated by plugging the optimal prices calculated using Corollary 1 into the profit function given by (1). This yields

Proposition 2 The optimal profit of a monopolist platform which operates on an asymmetric two-sided market and attracts $n_{i}$ number of agents on side $i$ is

$$
\pi=\frac{n_{1} c_{1}}{\eta_{1}-1}+\frac{n_{2} c_{2}}{\eta_{2}-1}-\frac{\eta_{1}}{\eta_{1}-1}\left[n_{1}\left(\alpha_{1} n_{1}+\alpha_{2} n_{2}\right)\right]
$$

Corollary $3 \frac{\partial \pi}{\partial \eta_{2}}<0 ; \frac{\partial \pi}{\partial \eta_{1}}>0$ if $\alpha_{1} n_{1}+\alpha_{2} n_{2}>c_{1}$, negative otherwise; $\frac{\partial \pi}{\partial \alpha_{i}}<0$ if $\eta_{1}>1$, positive otherwise.

The intuition behind Corollary 3 is that ceteris paribus the platform's ability to set higher prices to members of group 2 gets more and more limited as the group's demand is getting 
more elastic. However, a more elastic demand of group 1 can be beneficial if the total benefit a member of this group creates for the users is higher than the cost of serving this member. This is because if the demand of side 1 to join the platform is very elastic then a price drop for this group motivates the agents of this group to join the platform which induces the group members of other type to join the platform as well. And since the total benefit created by a joining member is larger than the costs to serve this member the platform pricing accordingly can increase its profit. Yet, ceteris paribus, a large external benefit created by a joining member of group 1 may hurt profits since in order to attract this member the platform should offer a discount equal to the benefit this member creates on the other users and as far as price discrimination is not allowed this discount is enjoyed by each member of group 1 .

In order to compare our results to the ones obtained in the case of standard symmetric two-sided markets by Armstrong (2006) let us assume symmetry in benefits an agent of type 1 enjoys by joining the platform, i.e., we assume that $\eta_{1}\left(p_{1} \mid n_{1}\right)=\eta_{1}\left(p_{1} \mid n_{2}\right)$ given that $n_{1}=n_{1}^{s}$ and $n_{2}=n_{2}^{s}$ agents join the platform. In this case we have that

$$
\begin{aligned}
& p_{1}=p_{1}^{s}-\frac{\eta_{1}}{\eta_{1}-1} \alpha_{1} n_{1} \\
& p_{2}=p_{2}^{s}+\frac{\eta_{2}}{\eta_{2}-1} \alpha_{1} n_{1}
\end{aligned}
$$

where $p_{1}^{s}, p_{2}^{s}$ are the monopoly prices in standard symmetric two-sided markets.

Corollary 4 If $\eta_{1}<1$ then $p_{i}>p_{i}^{s}$ for $i=1,2$, otherwise $p_{1}<p_{1}^{s}$ and $p_{2}>p_{2}^{s}$. If $\eta_{1}=\eta_{2}$ then $p_{1}+p_{2}=p_{1}^{s}+p_{2}^{s}$ and $p_{1}<p_{1}^{s}$ while $p_{2}>p_{2}^{s}$.

As one would expect the platform always sets a higher price to an agent whose presence does not elicit any external benefit. Yet, if an agent benefits only from the presence of its own type and the demand of this type is elastic the price set in the asymmetric case is lower then in the symmetric case. This is because in the asymmetric case the marginal benefit a member of group 1 creates on the platform is higher than in the symmetric case. Furthermore, since the benefits of joining the platform by a member of type 1 are solely influenced by the size of its own group, a price change on this side has larger effect relative to the symmetric case when members of type 1 benefits from the presence of the other group. Setting a high price for type 1 influences the size of group 2 which negatively affects the incentives for type 1 to join the platform. In other words, the platform is more limited in its pricing when benefits of joining the platform of a type 1 are affected by the size of the other group despite the fact that the demand is inelastic. Interestingly, if the price elasticities of demands are equal the total price $\left(p_{1}+p_{2}\right)$ set by a monopoly platform with asymmetric two-sided market equals the total price the monopolist would set in symmetric two-sided market, however, the price distribution among the two sides is different. Corollary 4 suggests that the platform with asymmetric two-sided market should subsidize agents whose presence creates utility on the other side of the market with a subsidy equal to the 
total benefit one agent may obtain from interactions. However, the optimal price strategy indicates that this subsidy is payed not by the platform, but by the agents from the other side of the market.

Comparing the profit a monopolist platform realizes in an asymmetric two-sided market to the profit achieved in the standard symmetric two-sided markets by Armstrong (2006) we have that

$$
\Delta \pi \equiv \pi-\pi^{s}=\alpha_{1} n_{1}\left(n_{2} \frac{\eta_{2}}{\eta_{2}-1}-n_{1} \frac{\eta_{1}}{\eta_{1}-1}\right)
$$

Corollary $5 \pi>\pi^{s}$ if the price elasticity of group 2 is small relative to the one of group 1 and/or the group of type 2 is significantly larger than the group of type 1.

The intuition behind this result is that in the asymmetric case the platform makes its profit mostly on group 2. Since the size of group 2 is not influencing the utility and consequently the willingness-to-pay of the group 1 the platform sets its price to group 2 solely based on the demand of group 2.

Let us now compare our results to the social optimum outcome. In order to do this we define total welfare as follows

\section{Definition 2}

$$
W\left(u_{1}, u_{2}\right) \equiv \pi\left(u_{1}, u_{2}\right)+\sum_{i=1}^{2} v_{i}\left(u_{i}\right)
$$

where $v_{i}\left(u_{i}\right)$ is the consumer surplus of group $i$ and satisfies the $v_{i}^{\prime}\left(u_{i}\right)=\Phi_{1}\left(u_{1}\right)$ condition for $i=1,2$.

Taking the first-order conditions of (3) with respect to utilities we have that the welfare maximizing outcome satisfies the following conditions

$$
\begin{aligned}
& \frac{\partial W\left(u_{1}, u_{2}\right)}{\partial u_{1}}=\Phi_{1}^{\prime}\left(u_{1}\right)\left[2 \alpha_{1} \Phi_{1}\left(u_{1}\right)+\alpha_{2} \Phi_{2}-u_{1}-c_{1}\right]=0 \\
& \frac{\partial W\left(u_{1}, u_{2}\right)}{\partial u_{2}}=\Phi_{2}^{\prime}\left(u_{2}\right)\left[\alpha_{2} \Phi_{1}\left(u_{1}\right)-u_{2}-c_{2}\right]=0
\end{aligned}
$$

Apart from the trivial solutions when $\Phi_{i}^{\prime}\left(u_{i}\right)=0$ for any $i=1,2$, we have that

Proposition 3 In the case of asymmetric two-sided markets the welfare maximizing prices satisfy

$$
\begin{aligned}
& p_{1}^{w}=c_{1}-\alpha_{1} n_{1}-\alpha_{2} n_{2} \\
& p_{2}^{w}=c_{2}
\end{aligned}
$$

and the optimal welfare equals to

$$
W^{\text {opt }}=n_{1}\left(\alpha_{1} n_{1}-c_{1}\right)+n_{2}\left(\alpha_{2} n_{1}-c_{2}\right)
$$


Proposition 3 suggests that in the welfare optimum agents of type 1 pay a price less than the cost of offering them the service and the difference equals the total benefit one agent creates for the other members using the platform.

Corollary 6 A profit maximizing monopoly platform always sets higher prices for each type of agent than the welfare maximizing prices, i.e. $p_{i}>p_{i}^{w}$ for every $i=1,2$.

\subsection{Competitive Platforms with Single-Homing}

In order to examine the optimal strategies of platforms in a competitive environment, let us introduce an other platform and assume that the two platforms, $A$ and $B$, compete with each other in a differentiated market. For the sake of simplicity we assume symmetric firms with the same costs functions, that is

Assumption $4 c_{i}^{A}=c_{i}^{B}=c_{i}$.

Agents' utilities, $u_{i}^{j}$ (where $i,=1,2$ and $j=A, B$ ), are determined in a similar way as it is assumed in Assumption 1, given that platform $j$ attracts $n_{i}^{j}$ number of members on side $i$ of the market and the prices set by platforms are $p_{i}^{j}$. More specifically

$$
\begin{array}{rlrl}
u_{1}^{A}=\alpha_{1} n_{1}^{A}-p_{1}^{A} ; & u_{2}^{A}=\alpha_{2} n_{1}^{A}-p_{2}^{A} \\
u_{1}^{B}=\alpha_{1} n_{1}^{B}-p_{1}^{B} ; & & u_{2}^{B}=\alpha_{2} n_{1}^{B}-p_{2}^{B}
\end{array}
$$

However, in order to consume each agent has to travel to a platform and by doing this faces a transportation cost represented by $t_{i}>0$. One may think of $t_{i}$ as the measure of product differentiation on side $i$ of the market, with $t_{i}$ being close to zero we assume that platforms offer homogeneous services, while with large $t_{i}$ we assume that platforms effectively serve different markets. We assume that agents are uniformly distributed along a unit line with the two platforms located at the end-points. That is, each agent is identified by a point on the unit line and this point corresponds to her most preferred brand. Furthermore, we assume, that on each side of the market agents join only one platform (known in the literature as single-homing) and in equilibrium each agent realizes positive utility, i.e., every agent is willing to join a platform. More formally

Assumption $5 \alpha_{2} \geq \max \left\{c_{1}+\frac{3}{2}\left(t_{1}-\alpha_{1}\right), 2 c_{2}+3 t_{2}\right\}$

The first part of the assumption ensures that agents of type 1 realize non-negative utility in equilibrium, while the second part is needed for the same reason in case of agents of type $2 .^{4}$

\footnotetext{
${ }^{4}$ Alternatively, this assumption could be substituted with an assumption about the existence of a large intrinsic value, $v_{i}^{0}$, an agent of type $i=1,2$ receives by joining a platform, as is in e.g. Armstrong and Wright (2006).
} 
The total number of agents of type $i$ a platform serves is determined by the location of the agent who is indifferent between joining platform $A$ or $B$, given that platforms offer $\left\{u_{i}^{A}, u_{i}^{B}\right\}$ utilities. That is, agents situated on the left-hand side of the indifferent consumer join platform $A$, while agents on the right-hand side join platform $B$. Denoting the indifferent agent's location by $\tilde{x}_{i}$ we have that

$$
u_{i}^{A}-t_{i} \tilde{x}_{i}=u_{i}^{B}-t_{i}\left(1-\tilde{x}_{i}\right)
$$

from which

$$
\tilde{x}_{i}=\frac{1}{2}+\frac{u_{i}^{A}-u_{i}^{B}}{2 t_{i}}
$$

Since the market is fully covered, we have that $n_{i}^{A}=\tilde{x_{i}}$ and $n_{i}^{B}=1-\tilde{x}_{i}$. Using (4) and (5), the market shares can be given with the following implicit functions

$$
n_{1}^{A}=\frac{1}{2}+\frac{\alpha_{1}\left(2 n_{1}^{A}-1\right)-\left(p_{1}^{A}-p_{1}^{B}\right)}{2 t_{1}} ; \quad n_{1}^{B}=1-n_{1}^{A}
$$

and

$$
n_{2}^{A}=\frac{1}{2}+\frac{\alpha_{2}\left(2 n_{1}^{A}-1\right)-\left(p_{2}^{A}-p_{2}^{B}\right)}{2 t_{2}} \quad n_{2}^{B}=1-n_{2}^{A}
$$

Solving the equation system given by (6) and (7) yields that the market shares of each platform, given that platform $A$ and $B$ set $\left\{p_{1}^{A}, p_{2}^{A}\right\}$ and $\left\{p_{1}^{B}, p_{2}^{B}\right\}$, respectively, are as follows

$$
\begin{aligned}
n_{1}^{A} & =\frac{1}{2}-\frac{p_{1}^{A}-p_{1}^{B}}{2\left(t_{1}-\alpha_{1}\right)} \\
n_{2}^{A} & =\frac{1}{2}-\frac{\alpha_{2}\left(p_{1}^{A}-p_{1}^{B}\right)}{2 t_{2}\left(t_{1}-\alpha_{1}\right)}-\frac{p_{2}^{A}-p_{2}^{B}}{2 t_{2}}
\end{aligned}
$$

and

$$
\begin{aligned}
& n_{1}^{B}=1-n_{1}^{A} \\
& n_{2}^{B}=1-n_{2}^{A}
\end{aligned}
$$

Platforms' profits are calculated as

$$
\pi^{j}=n_{1}^{j}\left(p_{1}^{j}-c_{1}\right)+n_{2}^{j}\left(p_{2}^{j}-c_{2}\right) \quad \text { for } \quad j=A, B
$$

The necessary and sufficient assumption for the concavity of profit functions is the following Assumption $6 t_{1}-\alpha_{1}>\frac{\alpha_{2}^{2}}{4 t_{2}}$ 
The same assumption is needed for the existence of market-sharing equilibria, in which, each platform makes at least non-negative profit.

Plugging into (10) the equilibrium market shares given by (8) and (9) we have that the profit maximizing prices satisfy

$$
\begin{aligned}
\frac{\partial \pi^{A}}{\partial p_{1}^{A}} & =\frac{\alpha_{2}\left(p_{2}^{A}-c_{2}\right)-t_{2}\left(t_{1}-\alpha_{1}+c_{1}-2 p_{1}^{A}+p_{1}^{B}\right)}{2 t_{2}\left(t_{1}-\alpha_{1}\right)}=0 \\
\frac{\partial \pi^{A}}{\partial p_{2}^{A}} & =\frac{\alpha_{2}\left(p_{1}^{A}-p_{1}^{B}\right)-\left(t_{1}-\alpha_{1}\right)\left(t_{2}+c_{2}-2 p_{2}^{A}+p_{2}^{B}\right)}{2 t_{2}\left(t_{1}-\alpha_{1}\right)}=0 \\
\frac{\partial \pi^{B}}{\partial p_{1}^{B}} & =\frac{\alpha_{2}\left(p_{2}^{B}-c_{2}\right)+t_{2}\left(\alpha_{1}-c_{1}-t_{1}-p_{1}^{A}+2 p_{1}^{B}\right)}{2 t_{2}\left(t_{1}-\alpha_{1}\right)}=0 \\
\frac{\partial \pi^{B}}{\partial p_{2}^{B}} & =\frac{\alpha_{2}\left(p_{1}^{B}-p_{1}^{A}\right)+\left(t_{1}-\alpha_{1}\right)\left[t_{2}(-1)-c_{2}-p_{2}^{A}+2 p_{2}^{B}\right]}{2 t_{2}\left(t_{1}-\alpha_{1}\right)}=0
\end{aligned}
$$

which leads to

Proposition 4 Symmetric platforms operating on an asymmetric two-sided markets serve half the agents of each type, set prices as

$$
\begin{aligned}
& p_{1}^{A}=c_{1}+t_{1}-\alpha_{1}-\alpha_{2} \\
& p_{1}^{B}=c_{1}+t_{1}-\alpha_{1}-\alpha_{2} \\
& p_{2}^{A}=c_{2}+t_{2} \\
& p_{2}^{B}=c_{2}+t_{2}
\end{aligned}
$$

and make profit of

$$
\pi^{j}=\frac{t_{1}+t_{2}-\alpha_{1}-\alpha_{2}}{2}
$$

for $j=A, B$.

Hence, platforms charge the agents of type 1 a price which equals with the costs adjusted downward with the value of benefits an agent creates for other users. Prices charged to agents of type 2 exhibit the same pattern as prices charged to type 1 , however, without the discount due to the benefits created for other users. Notice, that Assumption 6 guaranties that profits are positive in equilibrium. Furthermore, as we can see from the equilibrium profits network effects negatively affect firms' profits. The reason is that with external benefits created by users platforms have an additional reason to compete harder which at the end hurts profits.

Rearranging formulas describing the optimum prices and using the price elasticities of demand, which equal $\eta_{i}=\frac{p_{i}}{t_{i}}$ in Hotelling specification given firms symmetric locations and equal market-shares, we have that 


\section{Corollary 7}

$$
\begin{aligned}
\frac{p_{1}-c_{1}}{p_{1}} & =\frac{1}{\eta_{1}}-\frac{2\left(\alpha_{1} n_{1}+\alpha_{2} n_{2}\right)}{p_{1}} \\
\frac{p_{2}-c_{2}}{p_{2}} & =\frac{1}{\eta_{2}}
\end{aligned}
$$

Corollary 1 and 7 implies that competing platforms consider the external benefit created by a user of type 1 with a higher weight in their pricing strategies than a monopolist. This is because an addition user of type 1 makes the platform more attractive not just for the new joining members, but also for the users who are using the other platform. As we can see from the expressions competition takes place fully on the type 1's side of the market and on the other side platforms even though they perform in a competing environment still price as a monopoly platform would do. As the number of users joining the platforms' side 1 determines the demand for side 2 , after competition evolves on side 1 platforms can act as monopolist on side 2 .

Corollary $8 p_{2}^{j}>p_{1}^{j}$ for every $j=A, B$ whenever $c_{1}+t_{1}<c_{2}+t_{2}-\alpha_{1}-\alpha_{2}$

Corollary 8 suggests that if the firms locations are symmetric the prices charged to group 2 are always greater than prices set to agents of type 1 if the difference in costs of serving a type 1 relative to type 2 is not greater than the value of benefits these agents create. In other words, platforms charge the group of type 1 more aggressively if the benefits caused by the group are large or/and the brand differentiation is less relative to market side with group 2 agents.

Corollary $9 p_{1}^{j}=p_{1}^{j, s}-\alpha_{1}$ and $p_{2}^{j}=p_{2}^{j, s}+\alpha_{1}$, where $p_{2}^{j, s}$ stands for the equilibrium prices set by platform $j=A, B$ on symmetric two-sided-markets with platforms' locations at the end-points.

As we can see a similar pattern evolves here as in the monopoly case. Even though the total price set by a duopolist is similar to the price set by a monopoly, the price distribution is different. In order to attract agents of type 1 platforms set a lower price on this side of the market and charge a higher price equal to the discount a member of group 1 enjoys to the other side.

Corollary $10 \pi^{j}=\pi^{j, s}$ for every $j=A, B$, where $\pi^{j, s}$ stands for the profit earned by platform $j$ operating on a symmetric two-sided markets.

\subsection{Competitive Platforms with Partial Multi-Homing}

So far we assumed that each agent of type $i$ is able to join only one platform. Now, we extend our model to the case when agents of type 2 can join any of the platforms if they decide to. As agents of type 1 consider brand differentiation more relevant than network externalities, 
as assumed by Assumption 6, they tend to single-home, which gives an incentive to agents of type 2 to multi-home in order to maximize their external benefit. This is because users of type 2 solely benefit from the presence of the other type of agents no matter which platform they use. On the other hand, it is not rational to multi-home on side 1. This is because, if everyone on the side 1 would multi-home any agent of type 1 would have an incentive to join only one platform.

To determine profits maximizing prices each platform charges in this case, first consider the market size each platform serves. Since agents of type 1 single-home the analysis relevant to this side of the market presented in the previous section still holds. However, as agents of type 2 can multi-home the assumption of $n_{2}^{B}=1-n_{2}^{A}$ is no longer valid. To determine the demand each platform faces consider the following. If an agent of type 2 located at $x$ joins platform $A$ receives a utility of $u_{2}^{B}-t_{2} x$, while if she joins platform $B$ her utility is $u_{2}^{A}-t_{2}(1-x)$. If, however, this agent multi-homes and joins both platforms her utility is $u_{2}^{A}-t_{2} x+u_{2}^{B}-t_{2}(1-x)$. Thus, each agent with location characteristics for which $u_{2}^{A}-t_{2} x+u_{2}^{B}-t_{2}(1-x) \geq u_{2}^{A}-t_{2} x$ is satisfied prefers to multi-home rather than to single-home platform $A$. That is, given platforms' prices, the indifferent agent's location $\left(\tilde{x}_{2}^{A}\right)$ satisfies

$$
u_{2}^{A}-t_{2} \tilde{x}_{2}^{A}+u_{2}^{B}-t_{2}\left(1-\tilde{x}_{2}^{A}\right)=u_{2}^{A}-t_{2} \tilde{x}_{2}^{A}
$$

Using (4) this can be rewritten as

$$
\tilde{x}_{2}^{A}=1-\frac{\alpha_{2} n_{1}^{B}-p_{2}^{B}}{t_{2}}
$$

Similarly, the agent's location $\left(\tilde{x}_{2}^{B}\right)$ who is indifferent between single-homing platform $B$ or multi-homing both platforms can be given as

$$
\tilde{x}_{2}^{B}=\frac{\alpha_{2} n_{1}^{A}-p_{2}^{A}}{t_{2}}
$$

Hence, agents with location $x<\tilde{x}_{2}^{B}$ join platform $A$ and agents with location $x>\tilde{x}_{2}^{A}$ join platform $B$ as it is shown on Figure 1.

The following assumption ensures that agents of any type receive non-negative utilities in equilibrium.

Assumption $7 \quad c_{1} \leq \min \left\{\frac{\alpha_{2}}{4 t_{2}}\left(\alpha_{2}-2 c_{2}\right)-\frac{3}{2}\left(t_{1}-\alpha_{1}\right), \frac{\alpha_{2}}{2 t_{2}}\left(\alpha_{2}-2 c_{2}-2 t_{2}\right)-\frac{3}{2}\left(t_{1}-\alpha_{1}\right)\right\}$

Furthermore, Assumption 8 guaranties that platforms are willing to serve agents who join their market and make non-negative profits in equilibrium.

Assumption $8 t_{1}-\alpha_{1}>\max \left\{\frac{\alpha_{2}^{2}}{8 t_{2}}, \frac{\alpha_{2}}{2 z}\left(\alpha_{2}-2 c_{2}\right)-2\left(\alpha_{2}-c_{2}-t_{2}\right)\right\}$ 


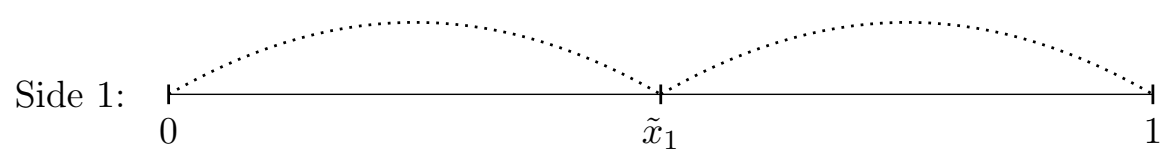

Side 2:

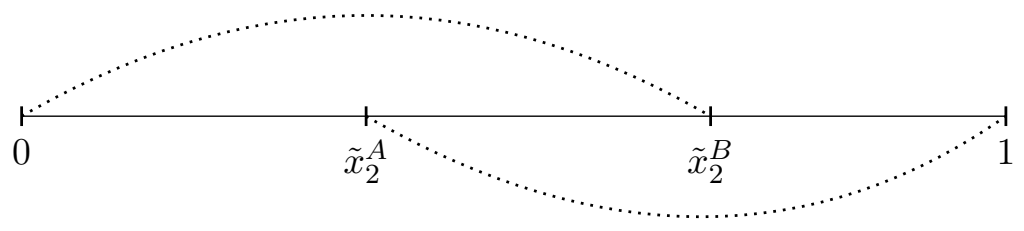

Figure 1: Location of indifferent agents and demands

From (6), (11) and (12) it follows that the demands platforms face on each side of the market, given that platforms set prices $\left\{p_{1}^{A}, p_{2}^{A}\right\}$ and $\left\{p_{1}^{B}, p_{2}^{B}\right\}$, respectively, are as follows

$$
\begin{aligned}
n_{1}^{A} & =\frac{1}{2}-\frac{p_{1}^{A}-p_{1}^{B}}{2\left(t_{1}-\alpha_{1}\right)} \\
n_{1}^{B} & =\frac{1}{2}-\frac{p_{1}^{B}-p_{1}^{A}}{2\left(t_{1}-\alpha_{1}\right)} \\
n_{2}^{A} & =\frac{\alpha_{2}}{2 t_{2}}-\frac{p_{1}^{A}-p_{1}^{B}}{2 t_{2}\left(t_{1}-\alpha_{1}\right)}-\frac{p_{2}^{A}}{t_{2}} \\
n_{2}^{B} & =\frac{\alpha_{2}}{2 t_{2}}-\frac{p_{1}^{B}-p_{1}^{A}}{2 t_{2}\left(t_{1}-\alpha_{1}\right)}-\frac{p_{2}^{B}}{t_{2}}
\end{aligned}
$$

Plugging these market shares into the profit functions given by (10) yields the following Proposition 5 Profit maximizing symmetric platforms operating on an asymmetric twosided market with partial multi-homing in equilibrium

i) if $\alpha_{2} \leq 4 t_{2}+2 c_{2}$, serve half the agents of type 1 and a measure of $\frac{\alpha_{2}-2 c_{2}}{4 t_{2}}$ agents of type 2 , set prices as

$$
\begin{aligned}
p_{1}^{A} & =c_{1}+t_{1}-\alpha_{1}-\frac{\alpha_{2}\left(\alpha_{2}-2 c_{2}\right)}{4 t_{2}} \\
p_{1}^{B} & =c_{1}+t_{1}-\alpha_{1}-\frac{\alpha_{2}\left(\alpha_{2}-2 c_{2}\right)}{4 t_{2}} \\
p_{2}^{A} & =\frac{\alpha_{2}+2 c_{2}}{4} \\
p_{2}^{B} & =\frac{\alpha_{2}+2 c_{2}}{4}
\end{aligned}
$$

and make profits equal to

$$
\pi^{j}=\frac{t_{1}-\alpha_{1}}{2}-\frac{\alpha_{2}^{2}-4 c_{2}^{2}}{16 t_{2}}
$$


ii) if $\alpha_{2}>4 t_{2}+2 c_{2}$, serve half the agents of type 1 and everyone from group 2 , set prices as

$$
\begin{aligned}
p_{1}^{A} & =c_{1}+t_{1}-\alpha_{1}-\frac{\alpha_{2}\left(\alpha_{2}-2 c_{2}-2 t_{2}\right)}{2 t_{2}} \\
p_{1}^{B} & =c_{1}+t_{1}-\alpha_{1}-\frac{\alpha_{2}\left(\alpha_{2}-2 c_{2}-2 t_{2}\right)}{2 t_{2}} \\
p_{2}^{A} & =\frac{\alpha_{2}-2 t_{2}}{2} \\
p_{2}^{B} & =\frac{\alpha_{2}-2 t_{2}}{2}
\end{aligned}
$$

and make profits equal to

$$
\pi^{j}=\frac{t_{1}-\alpha_{1}}{2}+\alpha_{2}-t_{2}-c_{2}-\frac{\alpha_{2}\left(\alpha_{2}-2 c_{2}\right)}{4 t_{2}}
$$

for $j=A, B$.

If $\alpha_{2} \leq 4 t_{2}+2 c_{2}$ the benefit an agent of type 2 receives by the presence of type 1 is not large enough to compensate the agent with the longest distance from the platform for traveling costs, i.e., agents with the highest transportation costs do not want to join the platform in question. In this case platforms' profit functions are concave in their own prices and the equation system defined by the first-order conditions determine the unconstrained solution given by Proposition 5. However, if the benefit created by type 1 agents are large enough, i.e. $\alpha_{2}>4 t_{2}+2 c_{2}$, each agent of type 2 wants to join both platforms and prefers multi-homing rather then single-home a platform. In this case, the platforms' problem is a constrained maximization problem where the constraints are given by the non-negative utility criteria for the furthest agents from the platforms.

Corollary $11 p_{1}^{j}<p_{2}^{j}$ for $j=A, B$.

As one would expect prices on side 2 are always higher than prices set on side 1 . However, Corollary 12 if $\alpha_{2} \leq 4 t_{2}+2 c_{2}$ then $p_{1}^{j} \geq p_{1}^{j, s h}$ and $p_{2}^{j} \leq p_{2}^{j, s h}$, for $j=A, B$, where $p_{i}^{j, s h}$ stands for the equilibrium price platform $j$ sets for side $i$ in case of single-homing, given by Proposition 4. Moreover, if $\alpha_{2}>4 t_{2}+2 c_{2}$ then $p_{1}^{j}<p_{1}^{j, s h}$ and $p_{2}^{j}>p_{2}^{j, s h}$.

The intuition behind Corollary 12 is that when the benefits agents of type 2 receive from the interactions with agents of type 1 is small then platforms compete more intensely in order to capture as large market-share as possible on side 2. This intensifies the direct competition on side 2 and softens the indirect competition on side 1 . As a result prices on side 1 increase and prices on side 2 decrease relative to the single-homing case. On the other hand, a large benefit agents of type 2 receive from the presence of type 1 makes multi-homing attractive. Platforms knowing that agents of type 2 will join both platforms, they will set prices accordingly and use limit-pricing. This, however, puts more pressure on the competition on side 1 , which decreases prices relative to the single-homing case. 
Corollary $13 \pi^{j, s h}>\pi^{j, m h}$ if $\alpha_{2}>(3+\sqrt{3}) t_{2}$ and $c_{2}<\frac{\alpha_{2}-2 t_{2}}{2}-\frac{t_{2}\left(\alpha_{2}-t_{2}\right)}{\alpha_{2}-2 t_{2}}$ for $j=A, B$, where $\pi^{j, s h}$ stands for the profits earned if all agents are single-homing, while $\pi^{j, m h}$ denotes the profit when multi-homing is allowed.

This can happen when the benefit created by agents of side 1 on side 2 is sufficiently large, and as a consequence all agents of type 2 want to multi-home, and to serve them involves small costs. The reason is that in this case platforms are involved in such a fierce competition on side 1 , that prices are driven down to zero or it even may result in negative prices (if negative prices are feasible) which eventually hurts profits. This result suggests that in some cases platforms may have strong incentives to deter agents from multi-homing. One way to achieve this is by imposing restriction on multi-homing for example with introduction of exclusive contracts.

Corollary $14 \frac{\partial p_{1}^{j}}{\partial \alpha_{i}}<0 ; \frac{\partial p_{2}^{j}}{\partial \alpha_{2}}>0$ and $\frac{\partial \pi^{j}}{\partial \alpha_{i}}<0$ for $i=1,2$ and $j=A, B$.

Equilibrium prices on side 1 are decreasing in network effects, however, on side 2 prices are increasing in them. Surprisingly, profits are negatively affected by the increase of these benefits. The intuition behind this is that as benefits on side 2 are increasing the prices on side 1 are decreasing since on side 1 prices are more sensitive for a change in benefits realized by agents on type 2 than they are on side 2 . Therefore, platforms need to offer a greater subsidy for agents on side 1 than they would with a lower network externality.

\section{References}

Armstrong, M (2006), 'Competition in two-sided markets', The RAND Journal of Economics, 37(3), pp. 668-691.

Armstrong, M. and Wright, J. (2006), 'Two-sided Markets, Competitive Bottlenecks and Exclusive Contracts', Economic Theory, 32(2), pp 353-380.

Hagiu, A. and Halaburda, H. (2014), 'Information and two-sided platform profits', International Journal of Industrial Organization, 34, pp. 25-35.

Rochet, J. C. and Tirole, J. (2003), 'Platform competition in two-sided markets', Journal of the European Economic Association, 1(4), pp. 990-1029.

Weyl, E. G. (2010), 'A Price Theory of Multi-Sided Platforms', American Economic Review, 100, pp 1642-1672. 